\title{
Don't just do something, stand there! The value and art of deliberate clinical inertia
}

\author{
Gerben KEIJZERS (10, ${ }^{1,2,3}$ Louise CULLEN ${ }^{10},{ }^{4}$ Diana EGERTON-WARBURTON ${ }^{5}$ and \\ Daniel M FATOVICH (10,7
}

\begin{abstract}
${ }^{1}$ Department of Emergency Medicine, Gold Coast University Hospital, Gold Coast, Queensland, Australia, ${ }^{2}$ School of Medicine, Bond University, Gold Coast, Queensland, Australia, ${ }^{3}$ School of Medicine, Griffith University, Gold Coast, Queensland, Australia, ${ }^{4}$ Emergency and Trauma Centre, Royal Brisbane and Women's Hospital, Queensland University of Technology, The University of Queensland, Brisbane, Queensland, Australia, ${ }^{5}$ School of Clinical Science at Monash Health, Monash University Faculty of Medicine, Nursing and Health Sciences, Melbourne, Victoria, Australia, ${ }^{6}$ Emergency Medicine, Royal Perth Hospital, The University of Western Australia, Perth, Western Australia, Australia, and ${ }^{7}$ Centre for Clinical Research in Emergency Medicine, Harry Perkins Institute of Medical Research, Perth, Western Australia, Australia
\end{abstract}

\begin{abstract}
It can be difficult to avoid unnecessary investigations and treatments, which are a form of low-value care. Yet every intervention in medicine has potential harms, which may outweigh the potential benefits. Deliberate clinical inertia is the art of doing nothing as a positive response. This paper provides suggestions on how to incorporate deliberate clinical inertia into our daily clinical practice, and gives an overview of current initiatives such as 'Choosing Wisely' and the 'Right Care Alliance'. The decision to 'do nothing' can be complex due to competing factors, and barriers to implementation are highlighted. Several strategies to promote deliberate clinical inertia are outlined, with an emphasis on shared decision-making. Preventing medical harm must become one of the pillars of modern health care and the art of not intervening, that is, deliberate clinical inertia, can be a novel patient-centred quality indicator to promote harm reduction.
\end{abstract}

Key words: Bayesian, clinical judgement, low-value care, overdiagnosis, shared decision-making.

\section{Introduction}

This is the first of a three-part series, reflecting on our clinical practice and our propensity to do 'something' for our patients, be it requesting investigations or providing treatment. This paper will outline reasons why it is hard to find a balance in avoiding unnecessary investigations and treatments, give an overview of current initiatives encouraging appropriate deliberate clinical inertia (i.e. actively doing nothing as a positive response) and provide suggestions on how to achieve this equilibrium between under- and over-doing.

\section{Outline of the problem}

Very few situations in medicine require immediate action. ${ }^{1}$ Patients with a more severe spectrum of disease are more likely to benefit from treatment, as beautifully illustrated in the book Overdiagnosed. ${ }^{2}$ Every action although has potential harms, which need to be weighed against the potential benefits. In clinical practice, 'risk versus benefit' is usually considered in terms of missing a diagnosis rather than potential risks

Correspondence: Professor Gerben Keijzers, Department of Emergency Medicine, Gold Coast University Hospital, 1 Hospital Boulevard, Southport, QLD 4215, Australia. Email: gbjmkeijzers@hotmail.com

Gerben Keijzers, MSc (Biomed Health Sci), MBBS, FACEM, PhD, Emergency Physician; Louise Cullen, MMBS (Hon), FACEM, PhD, Emergency Physician; Diana Egerton-Warburton, MBBS, FACEM, MClinEpi, MPH, Emergency Physician; Daniel M Fatovich, MBBS, FACEM, PhD, Emergency Physician, Head.

Accepted 4 December 2017

of treatment, so a better approach to care may be to ask; 'Is this intervention more likely to cause harm than the underlying condition with its possible harm or risk?' There are many reasons why 'doing nothing' is difficult, ${ }^{3}$ but doing what we can to provide excellent care while preventing medical harm from unnecessary interventions must become one of the pillars of modern holistic healthcare. $^{4}$

Doctors have a bias to intervene (intervention bias), whether it is with drugs, investigations or procedures in situations when not intervening would be a reasonable alternative. ${ }^{5}$ As clinicians, although we understand that no test is perfect, it is difficult to practice with that foremost in our minds. Fuelled by the popular media and by previous medical experiences, patients tend to believe that investigations and treatment are always beneficial. Unfortunately, patients consistently overestimate the benefits and underestimate the harms of investigations and procedures. ${ }^{6}$ Similarly, doctors underestimate how often treatments have no meaningful effect. Adding to these challenges in clinical practice is the context of our decision-dense and time poor environment of the ED. ${ }^{7}$

Hence, it is no surprise that in a USA survey of emergency physicians, $85 \%$ believed too many diagnostic tests are ordered in their own EDs and $97 \%$ said almost a quarter of the advanced imaging studies they personally order is medically 
unnecessary. ${ }^{8}$ These results may be (partially) driven by a different legal framework in the USA and as such fear of litigation was considered an important contributor, although fear of missing a potentially serious albeit low-probability diagnosis also played a part.

\section{Current initiatives encouraging deliberate clinical inertia}

The 'Choosing Wisely' $(\mathrm{CW})$ initiative was launched to improve stewardship of resource use by encouraging patients and clinicians to discuss the appropriateness of common interventions. ${ }^{9} \mathrm{CW}$ has helped summarise areas of low-value care, and their recommendations are endorsed by the relevant professional bodies, including the Australasian College for Emergency Medicine. ${ }^{10}$ In theory, these recommendations should reduce cognitive load and be easily adhered to in clinical practice. The CW recommendations were developed by individual professional bodies in a silo fashion. This risks the publication of contradicting advice or lack of consensus or consistency for patients that move between these silos.

Studies have assessed the effect of some $\mathrm{CW}$ recommendations and these include examples where multimodal interventions (including education, electronic health record redesign and audit-feedback loops) have reduced the use of a particular intervention in a specific setting, such as a reduction in the number of blood tests or advanced imaging tests in USA EDs. ${ }^{11,12}$ However, a population level review of seven CW recommendations (which included relevant ED topics such as imaging for uncomplicated headache; low back pain imaging without red-flag conditions; use of antibiotics for acute sinusitis) showed that without specific targeted education or alternative intervention, there was little effect in most of the areas investigated. ${ }^{13}$ These results may reflect a complex web of causality, including cultural barriers and society's intolerance of uncertainty and error. ${ }^{14}$
In the USA less than half of clinicians have ever discussed low-value care with a patient. ${ }^{15}$ There is no data available for Australia and New Zealand, and data are required from future studies in this area to better inform an approach in our setting. Furthermore, there is little incentive for the individual clinician to adhere to the recommendations. Hence, some advocate for 'doing nothing' as a key performance indicator of clinical care. ${ }^{3}$ This could be a novel quality initiative with established benchmarks for certain interventions.

The Lown Institute in the USA supports the 'Right Care Alliance' (RCA). ${ }^{16}$ Their focus is equitable care, and explicitly aims to ensure every intervention is value-adding and aligned with patient priorities. ${ }^{17}$ However, there is a paucity of published data that have measured the effect of the RCA. Their organisational structure is designed to avoid professional silos and promote crossdisciplinary communication and cooperation.

\section{Barriers for successful implementation of deliberate clinical inertia}

There is increasing momentum and appetite to capitalise on the philosophy of both the CW and RCA initiatives. However, to achieve meaningful outcomes, the mismatch between good intentions and realworld clinical practice need to be addressed. It is possible that clinicians consider avoiding low-value care, especially when discussing with peers or reading literature about this, but then deviate from those intentions in practice. Potential reasons for this disconnect may include:

1. The decision to 'do nothing' can be complex with competing (clinical and non-clinical) factors. Decision density and decision fatigue may lead to prioritisation of information and in times of high cognitive load humans become less receptive to additional information even if this is important. ${ }^{18}$ Consequently passive (reflexive) decision-making occurs. Alternatively, some clinicians may actively choose to use investigations or treatments known to be of low value, but may do so as a part of groupthink conformity. Purposefully deviating from guidelines is likely to be less common. ${ }^{19}$ These two types of thinking have been formally described by Kahneman. ${ }^{20}$ Type 1: The intuitive/ reflexive system: involves automatic decision-making based on pattern recognition. It is fast and requires little effort. Type 2: The analytical/problem-solving system: involves stepping deeper into analysis of the details of a patient's presentation. Ideally it involves estimating pre-test probabilities, considering alternative diagnoses, diagnostic accuracy of investigations, downstream adverse effects and post-test probability.

2. Clinicians perceive risks for both patients and themselves if they provide care without investigations or treatment. "What if this person has a poor outcome because I did not perform the (low-yield) test?' or 'Will my professional standing suffer if I miss a serious diagnosis?' Anecdote is powerful, as our brains respond emotionally to stories. Most of us will recall that one patient who had an atypical presentation but ultimately was diagnosed with a rare diagnosis after investigations. Such experiences encourage low value investigations or treatments, but also transfer its risk to the patient, especially if no discussion takes place on the risks and harms of these interventions.

Our society, lead by the medical profession, does not have a universally acceptable miss rate for diagnosis and as such the incentive to over-investigate and over-treat is strong. Variable risk tolerance and experience of clinicians may lead to practice variation and discussing alternative investigations and treatment options with patients is paramount. We need to explicitly promote the inevitable failures or delays in clinical care and encourage the profession and the public to 
define acceptable rates for missed diagnoses and adverse outcomes. ${ }^{14}$

Doctor-patient interactions are complex and these also influence decision-making. The theoretical framework of both CW and RCA are generally accepted by the majority of clinicians, but often these same clinicians will resort to concessions to optimal care, as this is the path of least resistance. Investigations and treatments do not give a binary answer or outcome, but rather change probabilities of disease and outcomes. The quantification of that effect is important and if the change in post-test probability is unlikely to affect the subsequent action, it is unhelpful (such as ordering a ddimer in a high-risk patient). Similarly, certain therapies require treatment of dozens of patients to have one patient with a better outcome, whereas the numbers needed to harm are often forgotten in the equation. ${ }^{21}$ This is exacerbated by the reporting and use of relative numbers instead of absolute values (a $50 \%$ relative reduction in outcome where the baseline event rate was $1 \%$ equates to a less impressive absolute reduction of $0.5 \%$ ). The use of patient-friendly decision aids is a practical strategy that addresses this. $^{22}$

\section{Strategies to promote deliberate clinical inertia}

How do we teach ourselves, our trainees and our students the art of masterly inactivity? How do we encourage not ordering investigations or starting treatments that are unlikely to be helpful for patients? How do we communicate with patients and their families to reduce low-value care? Actively 'doing nothing' requires knowledge about the strength and direction of the evidence of the relevant medical problem, clinical experience and confidence, transparent communication with the patient and a fundamental focus on acting in the patient's best interest. Many of these components are skills that can be taught and promoted through behavioural techniques that make it easier to do the right thing and harder to do the wrong thing (Table 1). Behavioural change can eventually lead to cultural change.

\section{Teaching clinical reasoning - incorporating pre-test probability}

Most clinicians are keen to improve and practice certain clinical skills such as electrocardiograph interpretation or point of care ultrasound (POCUS). However, clinical reasoning is not well taught. Clinical reasoning requires an understanding of statistics, odds and a Bayesian approach. Although most clinicians understand all investigations have imperfect test characteristics (there are always false positives and false negatives), the application of this knowledge may be counterintuitive. As with any skill, the key to mastery

TABLE 1. Strategies to achieve deliberate clinical inertia

Teaching clinical reasoning - incorporating pre-test probability

Re-framing 'doing nothing' as 'doing something'

- Empathy and acknowledgement

- Symptom management

- Clinical observation

- Education about natural course of the condition

- Managing expectations (in context of patient values and concerns)

- Shared decision-making

Incentives and rewards

Communicating rather than 'doing' - shared decision-making

is deliberate and purposeful practice. ${ }^{23}$ Nobody starts out as a POCUS expert - it takes dedication and focused training. Unfortunately, clinical reasoning using Type 2 thinking can sometimes be replaced by predominantly Type 1 thinking and although pattern recognition is valuable, it can lead to cognitive biases.

Medical knowledge about the prevalence and likelihood of alternative diagnoses will inform the pretest probability for any given condition. If this information is not available, the test result may be harmful rather than helpful. It was Archie Cochrane who said 'Before ordering a test, decide what you will do if it is (i) positive or (ii) negative. If both answers are the same, do not take the test.' This is where clinical training should go beyond mechanical rule following to hone expert clinical judgement. Obtaining information (i.e. investigation results) in the absence of judgement will not work in the patient's best interest. Doctors may forget that the practice of evidence-based healthcare, that is clinical decision-making, is the integration of clinical expertise using the best available evidence in the context of the values of the individual patient. One problem in medical education is that we value certainty from our students and present them with black and white multiple-choice examinations that do not represent the grey of real life medicine. ${ }^{24}$

When caring for patients, most doctors feel compelled to do something. Often an over-reliance on technology and investigations tends to replace analytical thinking, at least until after the technology has been used. ${ }^{25}$ This intervention bias is part of the problem and a sustained cultural change is required to be effective in achieving deliberate clinical inertia. Many of us were taught that $80-90 \%$ of diagnoses can be made simply by an appropriate clinical assessment, i.e. history and examination. ${ }^{26}$ However, technological 'certainty' is appealing.

Some clinicians fear causing harm by omission rather than by commission (My consultant wants it [or so I believe], I better do it, just in case). 
So, how do we teach and convince peers, trainees and students as well as patients and the broader health system and society in general to adopt deliberate clinical inertia as best practice? Fundamentally, it must be about awareness and recognition that the core nature of being a doctor is to put the needs of our patients first. Scott et al. advocate for cognitive huddles to disclose missteps in decision-making induced by biases related to clinical and non-clinical factors, as well as the awareness and understanding of one's own thought process (meta-cognition), to promote this in practice. ${ }^{7}$

\section{Re-framing 'doing nothing' as 'doing something'}

Remember the last adult patient you saw who you thought had a mild-tomoderate viral illness. How often can any testing be avoided? No blood tests, no X-ray, no nasopharyngeal aspirate, nothing. It is essential to understand what the patient's concerns are ${ }^{27}$ Patients may exclaim 'They did nothing for me!', whereas in all likelihood, these patients were told they had a self-limiting illness that required time, symptomatic treatment and self-care. They may have even had their symptoms managed; the natural course of the illness explained and had a discussion about the lack of value of antibiotic use to treat a viral illness.

These conversations need to be sign-posted as active treatment. For patients presenting to the ED with symptoms that do not require investigations or other interventions, an explicit guide could emphasise the following proponents of 'doing nothing' as active treatments as part of excellent care: (i) empathy and acknowledgment, (ii) symptom management, (iii) clinical observation, (iv) education about likely diagnosis including the natural course of the condition, (v) managing expectations and (vi) shared decision-making (SDM; see Table 2 for case vignette).

\section{Incentives and rewards}

A culture that blames and shames clinicians for missing a potential diagnosis is misleading and unhelpful. There is a belief that more is better and knowledge is power. This culture is reinforced at an institutional level by highlighting missed diagnoses in morbidity and mortality meetings. Medical literature and media reporting exacerbates this by an overwhelming focus on the
TABLE 2. Re-framing doing nothing as doing something: case vignette

A 42-year-old man was referred by his general practitioner after a computed tomography (CT) scan identified an old crush fracture of L2, caused by a farming accident 10 years previously. A recent injury had exacerbated his problem. The patient was distressed to have been told that he now had a fractured L2, because his old injury was a crushed L2. He did not understand that a crushed L2 was the same as a fractured L2. The recent CT was unchanged from the one 10 years earlier. On examination, there was no spinal tenderness. He had focal tenderness over a rib, clinically consistent with a fractured rib being the cause of his symptoms. I reflected that a fractured rib is a real nuisance (empathy and acknowledgment) and recommended simple analgesia (symptom management). Then I explained the likely course of expected symptoms over the following weeks (clinical observation and explanation of the natural course) and symptoms were likely to be exacerbated by laughing, moving and coughing. I also outlined that pain out of proportion or breathlessness would be reasons to seek a medical review (managing expectations). We discussed the options for pain management and the impact the pain and pain medicine may have on his job as a carpenter. A shared decision was made to use over the counter analgesia only with a return to work based on symptoms (shared decision-making). benefits of a treatment, but not the harms. Some down-stream harms (e.g. antibiotic resistance, superinfection with Clostridium difficile, follow-up imaging and interventions for 'incidental findings', cost, time and anxiety) are not captured by current key performance indicators. This change in culture would be helped by an endorsed 'acceptable miss rate', which has been proposed for certain 'not to miss' diagnoses. ${ }^{28}$ This should be context-specific and as such will need ongoing involvement with patients and the community, supported through information sharing, evidence-based SDM and broad public engagement. ${ }^{29}$ Furthermore, work needs to be done to ensure clear benchmarks for acceptable miss rates by relevant institutions and organisations, including medical colleges.

\section{Communicating rather than doing - SDM}

A focus on the art of medicine and listening to patients will enhance diagnostic acumen with many diagnoses discernible from history alone. $^{26}$ Treatment decisions should actively include SDM, a process where doctors and patients collaboratively discuss potential management strategies when more than one reasonable option exists and together reach a decision based on both the available evidence and the patient's values and preferences. ${ }^{4,30}$ The $\mathrm{CW}$ campaign has encouraged increased involvement of the public in their care and the provision of relevant and understandable information to enable people to make informed choices. This has been aptly rephrased as 'Choosing Together', emphasising the important principles of patient-centred care. ${ }^{31,32}$ Aligning the interests of patients, clinicians, researchers and policymakers will lead to a more collaborative approach in sharing decisions about care, services and research. ${ }^{33}$

Clinical research into care incorporating SDM and decision aids in their design have shown a decrease in investigations and admissions. ${ }^{34,35}$ 
Doctors often assume patients want more investigations and treatments, but these studies show that when harms and benefits are described in a patient-friendly format, patients often choose the 'less is more' option.

\section{Conclusion}

There is increasing awareness that preventing medical harm must become one of the pillars of modern healthcare. Adopting deliberate clinical inertia, which is the art of not intervening, as a specific measurable indicator, would be a novel patientcentred quality initiative.

\section{Next chapter}

The next paper in this series will explore some common clinical conditions where deliberate clinical inertia can be employed to reduce low-value care and improve clinicians' and patients' experience with the healthcare system. These will include peripheral i.v. catheter insertion, renal colic and low risk chest pain investigation and management.

\section{Competing interests}

GK and DEW are section editors for Emergency Medicine Australasia.

\section{References}

1. Fatovich DM. The time paradox of emergency medicine: another inverted U curve. Emerg. Med. Australas. 2017; 29: 730-2.

2. Welch GG, Schwartzl L, Woloshin S. Overdiagnosed: Making People Sick in the Pursuit of Health, 1st edn. Boston, MA: Beacon Press, 2011.

3. Keijzers G. Critical thinking, curiosity and parsimony in (emergency) medicine: 'doing nothing' as a quality measure? Emerg. Med. Australas. 2017; 29: 360-2.

4. Hoffman JR, Wilkes MS. Don't just do something, stand there. Revista Brasileira de Medicina de Família e Comunidade 2015; 10: 1-2.

5. Foy AJ, Filippone EJ. The case for intervention bias in the practice of medicine. Yale J. Biol. Med. 2013; 86: 271-80.

6. Krouss M, Croft L, Morgan DJ. Physician understanding and ability to communicate harms and benefits of common medical treatments. JAMA Intern. Med. 2016; 176: 1565-7.

7. Scott IA, Soon J, Elshaug AG, Lindner R. Countering cognitive biases in minimising low value care. Med. J. Aust. 2017; 206: 407-11.

8. Kanzaria HK, Hoffman JR, Probst MA, Caloyeras JP, Berry SH, Brook RH. Emergency physician perceptions of medically unnecessary advanced diagnostic imaging. Acad. Emerg. Med. 2015; 22: 390-8.

9. Cassel CK, Guest JA. Choosing wisely: helping physicians and patients make smart decisions about their care. JAMA 2012; 307: 1801-2.

10. Australasian College for Emergency Medicine. Tests, Treatments and Procedures Clinicians and Consumers Should Question. [Cited 20 Oct 2017.] Available from URL: http://www.choosingwisely.org.au/ recommendations/acem

11. Venkatesh AK, Hajdasz D, Rothenberg $\mathrm{C}$ et al. Reducing unnecessary blood chemistry testing in the emergency department: implementation of choosing wisely. Am. J. Med. Qual. 2017. https:// doi.org/10.1177/1062860617691842

12. Raja AS, Venkatesh A, Mick N et al. 'Choosing wisely' imaging recommendations: initial implementation in New England emergency departments. West J. Emerg. Med. 2017; 18: 454-8.

13. Rosenberg A, Agiro A, Gottlieb M et al. Early trends among seven recommendations from the choosing wisely campaign. JAMA Intern. Med. 2015; 175: 1913-20.

14. Hoffman JR, Kanzaria HK. Intolerance of error and culture of blame drive medical excess. BMJ 2014; 349: g5702.

15. Maughan BC, Baren JM, Shea JA, Merchant RM. Choosing wisely in emergency medicine: a National Survey of emergency medicine academic chairs and division chiefs. Acad. Emerg. Med. 2015; 22: 1506-10.
16. The Right Care Alliance. [Cited 20 Oct 2017.] Available from URL: https://rightcarealliance.org

17. Carpenter CR, Raja AS, Brown MD. Overtesting and the downstream consequences of overtreatment: implications of 'preventing overdiagnosis' for emergency medicine. Acad. Emerg. Med. 2015; 22: 1484-92.

18. Sweller J. Cognitive load during problem solving: effects on learning. Cognit. Sci. 1988; 12: 257-85.

19. Lenzer J. Why we can't trust clinical guidelines. BMJ 2013; 346: f3830.

20. Kahneman D. Thinking Fast and Slow. New York, NY: Farrar, Strauss and Giroux, 2011.

21. The NNT. Quick Summaries of Evidence-Based Medicine. [Cited 21 Oct 2017.] Available from URL: www.thennt.com

22. Mayo Clinic Shared Decision Making National Resource Center. [Cited 1 Nov 2017.] Available from URL: http://shareddecisions. mayoclinic.org

23. Ericsson KA, Pool R. Peak. How to Master Almost Anything. Boston, MA: Houghton Mifflin Harcourt, 2016.

24. Wray CM, Loo LK. The diagnosis, prognosis, and treatment of medical uncertainty. J. Grad. Med. Educ. 2015; 7: 523-7.

25. Fatovich DM. The inverted U curve and emergency medicine: overdiagnosis and the law of unintended consequences. Emerg. Med. Australas. 2016; 28: 480-2.

26. Hampton JR, Harrison MJ, Mitchell JR, Prichard JS, Seymour C. Relative contributions of history-taking, physical examination, and laboratory investigation to diagnosis and management of medical outpatients. BMJ 1975; 2: 486-9.

27. van der Wel MC. First 'more', then 'less'. Arch. Intern. Med. 2012; 172: 465.

28. Pines JM, Szyld D. Risk tolerance for the exclusion of potentially lifethreatening diseases in the ED. Am. J. Emerg. Med. 2007; 25: 540-4.

29. Elshaug AG, Rosenthal MB, Lavis JN et al. Levers for addressing medical underuse and overuse: achieving high-value health care. Lancet 2017; 390: 191-202. 
30. Kanzaria HK, Brook RH, 32. Richards T, Coulter A, Wicks P. Probst MA, Harris D, Berry SH, Hoffman JR. Emergency physician perceptions of shared decision-making. Acad. Emerg. Med. 2015; 22: 399-405.

31. Rashid A. Choosing together: encouraging person centred care and shared decision making. BMJ 2015; 350: h2935.
Time to deliver patient centred care. BMJ 2015; 350: h530.

33. Keijzers G, Thom O, Taylor D, Knott J, Taylor DM, Clinical Trials Group, Australasian College for Emergency Medicine. Clinical research priorities in emergency medicine. Emerg. Med. Australas. 2014; 26: 19-27.
34. Hess EP, Hollander JE, Schaffer JT et al. Shared decision making in patients with low risk chest pain: prospective randomized pragmatic trial. BMJ 2016; 355: i6165.

35. Hess EP, Knoedler MA, Shah ND et al. The chest pain choice decision aid: a randomized trial. Circ. Cardiovasc. Qual. Outcomes 2012; 5: 251-9. 